\title{
Interaction Effects in the Mesoscopic Regime: A Quantum Monte Carlo Study of Irregular Quantum Dots
}

\author{
Amit Ghosal, ${ }^{1}$ C. J. Umrigar, ${ }^{2}$ Hong Jiang, 1,3 , Ð Denis Ullmo, ${ }^{1,}$ † and Harold U. Baranger ${ }^{1}$ \\ ${ }^{I}$ Department of Physics, Duke University, Durham, North Carolina 27708-0305 \\ ${ }^{2}$ Theory Center and Laboratory of Atomic and Solid State Physics, Cornell University, Ithaca, New York 14853 \\ ${ }^{3}$ Department of Chemistry, Duke University, Durham, North Carolina 27708-0354
}

(Dated: November 17, 2017)

\begin{abstract}
We address the issue of accurately treating interaction effects in the mesoscopic regime by investigating the ground state properties of isolated irregular quantum dots. Quantum Monte Carlo techniques are used to calculate the distributions of ground state spin and addition energy. We find a reduced probability of high spin and a somewhat larger even/odd alternation in the addition energy from quantum Monte Carlo than in local spin density functional theory. In both approaches, the even/odd effect gets smaller with increasing number of electrons, contrary to the theoretical understanding of large dots. We argue that the local spin density approximation over predicts the effects of interactions in quantum dots.
\end{abstract}

PACS numbers: 73.23.Hk, 73.63.Kv, 02.70.Ss

The interplay between correlations and quantum mechanical interference of electron states, long at center stage in condensed matter physics, has been traditionally investigated in disordered systems but can also be probed in confined systems, such as quantum dots ${ }^{1,2}$ In these latter, the confinement leads to mesoscopic fluctuations ${ }^{1.3}$ which in turn modify the role of Coulomb repulsion between electrons within the dots. Quantum dots offer the great practical advantage of experimental tunability in the study of this interplay. 1.3

Quantum dots of different size give rise naturally to different descriptions. For small dots, including both vertica ${ }^{4}$ and few electron latera $1^{\frac{5}{\underline{n}}}$ dots, circular symmetry is preserved and plays a critical role. In this limit quantum Monte Carlo (QMC) calculations have been performed ${ }^{6,7,8,9,10}$ as well as simpler density functional theory (DFT) simulations $\frac{11.12}{12}$ A comparative study for the weak interaction regime ${ }^{7.8}$ confirmed the validity of the DFT method in this small dot limit.

For large irregular dots, $, 1,3$ on the other hand, all spatial symmetries are broken. For a sufficiently irregular shape, the motion of electrons within the dot is chaotic, which then justifies modeling the single-particle energies and wave functions by random matrix theory and random plane waves, respectively 13,14 Furthermore, interaction effects in these larger dots are often treated within the random phase approximation (RPA) for gas parameter $r_{s}$ (the ratio between the interaction energy and kinetic energy, formally defined as $r_{s}=1 / \sqrt{\pi n} a_{0}$ for $2 \mathrm{D}$ bulk systems, which thus identifies the strength of the interaction) of order 1 or smaller ${ }^{2,15}$ The "universal Hamiltonian" picture ${ }^{2.16}$ that emerges leads to statistical predictions for various quantities, such as the ground

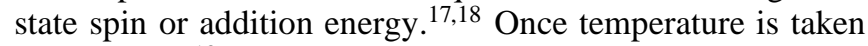
into account,, 18 these are in good agreement with experimental data ${ }^{19,20,21,22}$ One notable feature is a substantial difference at zero temperature between dots containing an odd number of electrons, $N$, and those in which this number is even. Experiments have not to date performed at a sufficiently low temperature to probe this feature. This even/odd effect persists for large $N$ with an essentially unchanged magnitude provided that $r_{s}$ remains constant.

In order to go beyond statistical predictions and address features of individual irregular dots, an approach which accurately treats the combination of mesoscopic fluctuations and interaction effects is needed. DFT appeared a natural choice for such studies, and, indeed, microscopic calculations of ground state energies for large irregular dots $(N \sim 200)$ were carried out within the framework of the local spin density approximation (LSDA) ${ }^{23,24,25}$ The statistics of the LSDA results turned out, however, to be in qualitative disagreement with the earlier predictions, even for the modest interaction strengths $\left(r_{s} \sim 1.5\right)$ that are experimentally relevant; for instance, in the LSDA results at zero temperature, the even/odd effect is nearly absent. In fact, there were several indications of stronger interactions in LSDA than those obtained from RPA. The striking discrepancy between the two approaches both of which are believed to be valid in the range of $r_{s}$ considered - combined with the absence of experimental statistics for low temperature keeps this problem open.

Here we take up the issue of accurately treating interaction effects in the mesoscopic regime. We consider irregular quantum dots with up to 30 electrons. The lack of symmetryinduced shell structure makes irregular dots qualitatively different from circular dots; in particular, the mesoscopic interference effects are both more subtle and more generic. In this regime, where the universal Hamiltonian picture is not expected to hold because of the modest size, we use QMC to treat the interactions much more carefully than in LSDA. To this end, we present QMC calculations of the addition energy and ground state spin for such dots, and compare to corresponding LSDA results.

We consider a model quantum dot consisting of electrons moving in a two dimensional plane, with kinetic energy $\left(-\frac{1}{2} \sum_{i} \nabla_{i}^{2}\right)$, and interacting with each other by long-range Coulomb repulsion $\left(\sum_{i<j}\left|\mathbf{r}_{i}-\mathbf{r}_{j}\right|^{-1}\right)$. All energies are expressed in atomic units, defined by $\hbar=e^{2} / \epsilon=m^{*}=1$, with electronic charge $e$, effective mass $m^{*}$, and dielectric constant of the medium $\epsilon$. The electrons are confined by an external (quartic) potential

$$
V_{\text {ext }}(x, y)=a\left[\frac{x^{4}}{b}+b y^{4}-2 \lambda x^{2} y^{2}+\gamma(x-y) x y r\right]
$$


where $r=\sqrt{x^{2}+y^{2}}$. This simple form of $V_{\text {ext }}$ breaks all symmetries except time reversal invariance. It leads to chaotic motion of the electrons inside the dot ${ }^{26}$ which is the experimental situation for large dots $\frac{1.19,20.21 .22}{1}$ in fact, not only the bare $V_{\text {ext }}$ but also the self-consistent potential leads to chaotic dynamics ${ }^{23,24}$ We have studied potential (1) for a range of parameters and report here results for $a=0.002$ (which controls $\left.r_{s}\right), b=\pi / 4, \gamma$ between 0.1 and 0.2 (break spatial symmetries), and $\lambda$ between 0.53 and 0.67. For these parameters, the dynamics in the bare potential is chaotic. We accumulate statistics for six dots formed by different sets of parameters $(\lambda$ and $\gamma$ ) from the above range. We study dots with $N=10$ to 30 electrons which yields a range $r_{s}=1.8$ to 1.32 .27

Variational (VMC) and diffusion (DMC) Monte Carlo techniques ${ }^{28}$ were used to calculate the energies $E(N, S)$ of our model quantum dots for each $N$ and spin $S$. We investigated $S=0,1$, and 2 for even $N$, and $S=1 / 2,3 / 2$, and $5 / 2$ for odd $N$. For a given $V_{\text {ext }}$, the ground state energy $E_{\mathrm{GS}}$ and the ground state spin $S_{\mathrm{GS}}$ were determined for each $N$.

The trial wave function used in $\mathrm{QMC}, \Psi_{T}$, is written as a linear combination of products of up- and down-spin Slater determinants multiplied by a Jastrow factor. Each Slater determinant is constructed from single-particle Kohn-Sham (KS) orbitals obtained using the LSDA functional. The Jastrow factor effectively describes the dynamic correlation between the electrons coming from their mutual repulsion, whereas the near-degeneracy or static correlation is taken into account by having more than one determinant. We optimize the Jastrow parameters and determinant coefficients by minimizing the variance of the local energy. ${ }^{29}$

In a second stage, we use fixed-node DMC ${ }^{28.30}$ to project the optimized many-body wavefunction onto a better approximation of the true ground state. The fixed-node DMC energy is an upper bound to the true energy and depends only on the nodes of the trial wave function, i.e., only on the linear combination of determinants. (The Jastrow factor affects the statistical error of the energy but not its expectation value.) The statistical error in the energy $E_{\mathrm{DMC}}(N, S)$ obtained in this way is smaller than the single-particle mean level spacing $\Delta$ by about two orders of magnitude and hence is insignificant. The systematic error from the fixed-node approximation of the many-body wave function is, however, difficult to estimate though experience suggests it is often small. We have included Slater determinants for which the sum of the KS single-particle energies are up to $\Delta$ greater than the sum of the KS single-particle energies for the ground state KS determinant. This amounts to taking mostly one and sometimes two or three Slater determinants in the $\Psi_{T}$ expansion. Increasing the energy window from $\Delta$ to $3 \Delta$ failed to reduce $E_{\mathrm{DMC}}$ although it sometimes reduced $E_{\mathrm{VMC}}$.

We present the distribution of $S_{\mathrm{GS}}$ obtained from both DMC and LSDA ${ }^{31}$ in Fig. 1(a). Within a model of effectively non-interacting electrons, $S_{\mathrm{GS}}$ is 0 or $1 / 2$ for even $N$ and odd $N$, respectively, due to standard up/down filling of the orbitals. We see that the probability of finding a non-trivial $S_{\mathrm{GS}}$, i.e. not zero or half, is substantial. Interestingly, this probability is reduced in DMC calculations compared to LSDA. Note that the differences between the two distributions, although
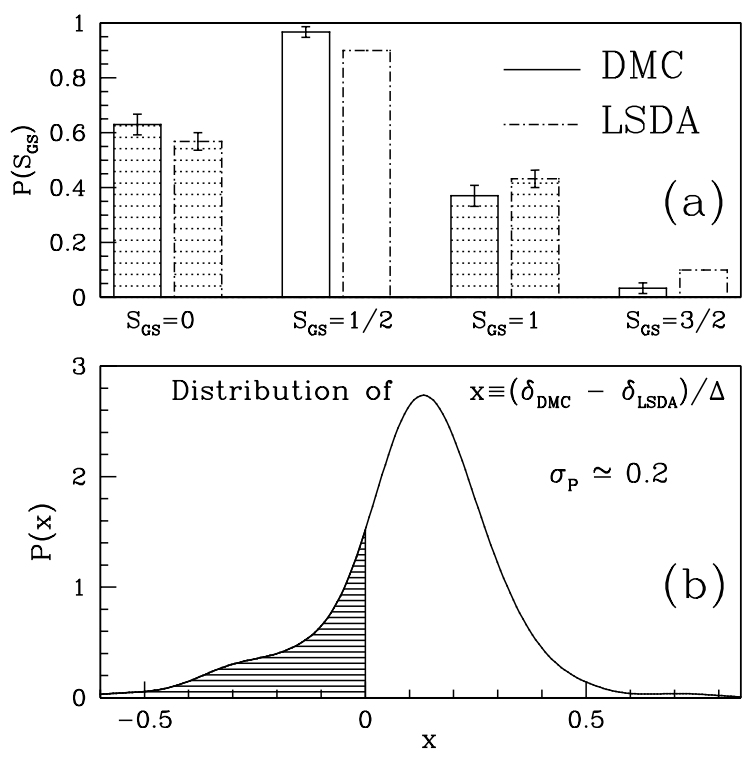

FIG. 1: (a) Distribution of $S_{\mathrm{GS}}$ from DMC and LSDA calculations. Shaded histograms are for dots with even $N$ while unfilled bars are for odd $N$; data is collected for $N=10-30$ and six realizations of $V_{\text {ext }}$, which are also used to estimate the statistical error. Though the differences are small, LSDA predicts a larger probability of nontrivial $S_{\text {GS. }}$ (b) Distribution of the difference in "spin gap" obtained using DMC and LSDA, normalized by the mean level spacing $\Delta$. The large width of the distribution $\left(\sigma_{P}\right)$ indicates a significant difference between the two techniques. Note that $x$ (defined in the figure) is primarily positive; negative values occur predominantly when the ground state has non-trivial spin. LSDA is, therefore, making nontrivial spin states more probable by lowering their energies compared to DMC results. [A sliding Gaussian window of width 0.08 is used to give a smooth estimate for $P(x)$.]

clearly visible, are not large and are therefore not much bigger than the statistical error given the relatively small data set $(21 \times 6=126$ cases total). There are, however, significant correlations between the LSDA and DMC results. In fact, $S_{\mathrm{GS}}$ from DMC is, up to one exception, always the same as or lower than that from LSDA. As a consequence the statistical error on $\left\langle S_{\mathrm{GS}}^{\mathrm{LSDA}}-S_{\mathrm{GS}}^{\mathrm{DMC}}\right\rangle$ is only $30 \%$ of its value. Thus, there is a clear difference between LSDA and DMC in the predicted ground state spin.

The ground state spin shows the difference between LSDA and DMC results at only a coarse level. To obtain a more detailed understanding, we focus on the "spin gap", $\delta$, which we define as $\delta=E(S=1)-E(S=0)$ for even $N$ and $\delta=$ $E(S=3 / 2)-E(S=1 / 2)$ for odd $N$. Thus $\delta$ is the amount by which the higher spin state differs in energy from the lower. Changing the spin of a dot from 0 or $1 / 2$ to a higher value involves a competition between the single-particle energy cost and the exchange energy, $-J S(S+1)$, gain ${ }^{32}$

The key result for comparing DMC and LSDA is shown in Fig. 1(b): the distribution of $\left(\delta_{\mathrm{DMC}}-\delta_{\mathrm{LSDA}}\right) / \Delta$. Note, first, that the distribution is broad (full width $\sim 0.4 \Delta$ ); it is of order the energy required to flip $S_{\mathrm{GS}}$ from 0 to 1 for an even dot, $\Delta-2 J$, assuming a realistic value of the exchange parameter 


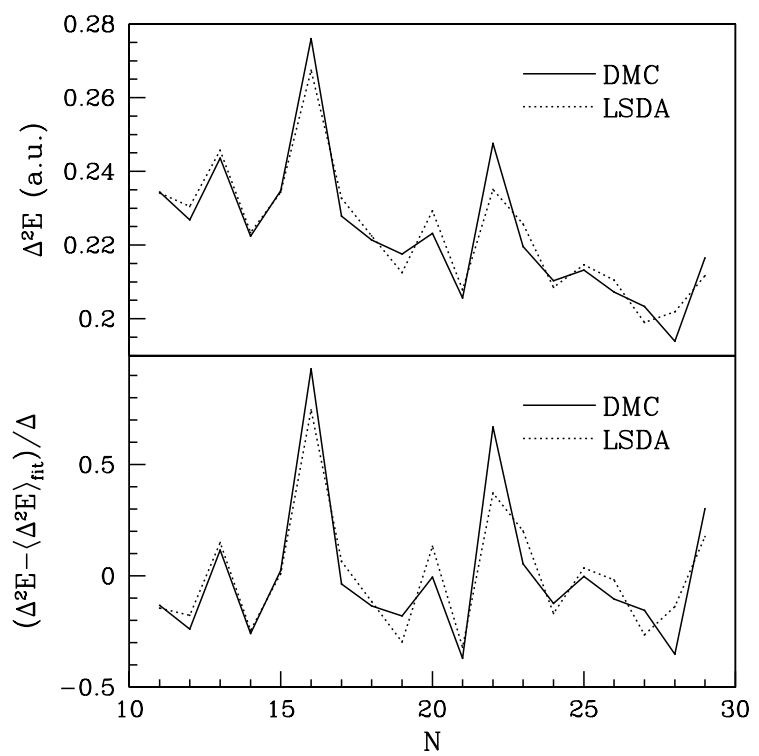

FIG. 2: Top: The addition energy from both DMC and LSDA for one realization of $V_{\text {ext }}$ as a function of the number of electrons on the dot. Note the large mesoscopic fluctuations in $\Delta^{2} E$ around the expected overall decrease. Bottom: Fluctuation in the addition energy after removing the smooth part, normalized to the mean level spacing $\Delta$. We see that the fluctuations are of order $\Delta$ and that they are somewhat larger for DMC results than for LSDA.

$J \sim 0.35$ for $r_{s} \sim 1.5$. Second, note that the spin gap in DMC tends to be larger than that in LSDA. This indicates that the strength of interactions in LSDA is overestimated. Finally, we have studied this quantity separately for the smaller $(N=10$ $20)$ and larger $(N=20-30)$ dots. We have not found any size dependence - results in both ranges of $N$ are the same as in Fig. 1(b) within our statistical accuracy. These observations, together with the results of Fig. 1(a), show that LSDA unduly favors non-trivial spin states.

The ground state spin distribution has implications for the distribution of the spacing between Coulomb blockade conductance peaks, ${ }^{17}$ through its relation to $E_{\mathrm{GS}}$. In the nearly isolated dot limit, the spacing between the Coulomb blockade conductance peaks is proportional to the the addition energy 1 defined by

$$
\Delta^{2} E(N)=E_{\mathrm{GS}}(N+1)+E_{\mathrm{GS}}(N-1)-2 E_{\mathrm{GS}}(N) .
$$

For noninteracting electrons one would have

$$
\Delta^{2} E(N)= \begin{cases}\epsilon_{N / 2}-\epsilon_{N / 2-1} & \text { for even } N \\ 0 & \text { for odd } N\end{cases}
$$

where $\epsilon_{i}$ are the energies of the single-particle states. Note the sharply different characteristics of even and odd $N$ dots. Interactions reduce this strong even/odd effect.

The behavior of $\Delta^{2} E(N)$ for a particular realization of $V_{\text {ext }}$ is presented in the top panel of Fig. 2. Similar qualitative behavior is observed for all other configurations we studied. An overall decrease of $\Delta^{2} E$ with $N$ is expected due to the increase of effective capacitance of the dot and, hence, the

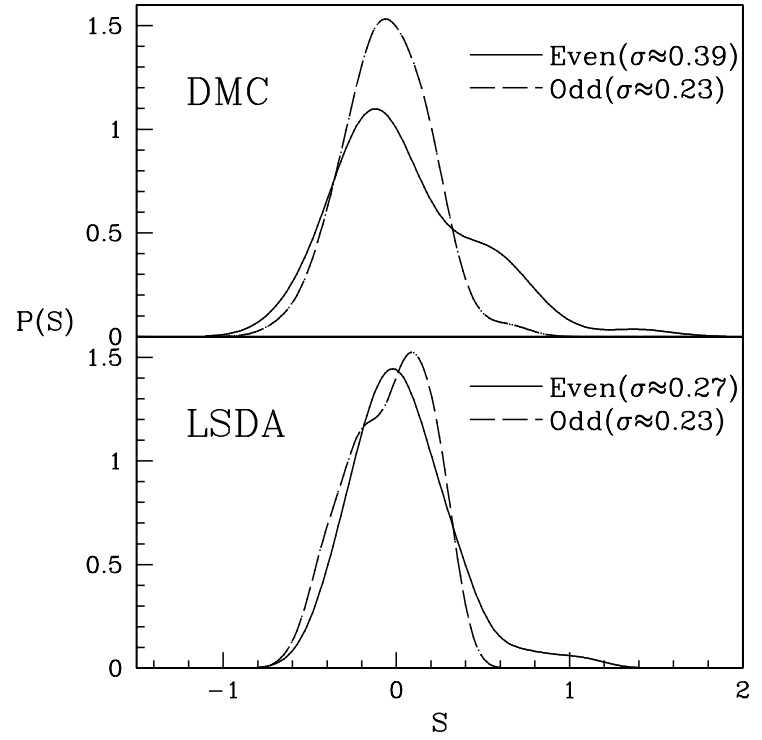

FIG. 3: Distribution of the normalized fluctuations in the conductance peak spacing, $s \equiv\left(\Delta^{2} E-\left\langle\Delta^{2} E\right\rangle_{\text {fit }}\right) / \Delta$ from DMC (top) and LSDA (bottom) calculations. The DMC distributions for even (solid) and odd (dashed) $N$ are quite different while there is less difference for LSDA. The standard deviation in the different cases, $\sigma$, quantifies this contrast. (A sliding Gaussian window of width 0.17 (for even) and 0.1 (for odd) is used to obtain a smooth curve.)

decrease in the classical charging energy as the dot gets bigger. On top of the mean behavior, we clearly see strong mesoscopic fluctuations arising from the interplay of electron interaction and interference effects in the irregular dots. The fluctuations seem to be slightly larger in the DMC results than in LSDA. To focus on these fluctuations, we subtract the smooth classical part using a linear fit, and present the fluctuating part normalized by the mean level spacing $\Delta$ (which is the natural scale of these mesoscopic fluctuations) in the lower panel.

To get a more quantitative picture, we plot the distribution of the normalized addition energy fluctuations in Fig. 3. Note the larger difference between the two distributions obtained from DMC than between those obtained from LSDA. In the DMC results, the width of the distribution for even $N$ is significantly larger than that for odd dots, and the even $N$ distribution has a long tail reminiscent of the Wigner surmise for the distribution of $\epsilon_{N / 2}-\epsilon_{N / 2-1}$ found using random matrix theory (RMT) [see Eq. [3] ].

Looking at the data for smaller and larger dots separately, we find a strong trend shown in Fig. 4: the even/odd effect in the DMC data decreases significantly as $N$ increases. If extrapolated to much larger $N$, this trend, also present in our LSDA results, contradicts the prediction ${ }^{17.18}$ of combining RPA interactions with an RMT treatment of the single particle statistics.

In conclusion, we have used quantum Monte Carlo to accurately investigate the role of interactions in the mesoscopic regime. We find that for irregular dots with a gas parameter $r_{s} \sim 1.5$ and electron number in the range 10 to $30, \mathrm{DMC}$ calculations show (1) mesoscopic fluctuations of the addition 


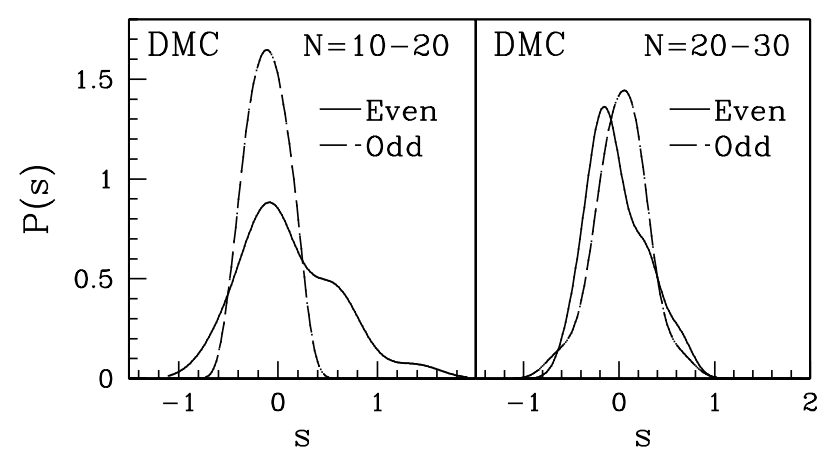

FIG. 4: Distribution of the normalized fluctuations in the conductance peak spacing, from DMC calculations in the range of $N=10$ 20 (left) and $N=20-30$ (right). For smaller $N$, the even/odd effect is stronger: $\sigma_{\text {even }}=0.46 \pm 0.08$, while $\sigma_{\text {odd }}=0.18 \pm 0.02$. On the other hand for larger $N$, the even/odd effect is significantly reduced: $\sigma_{\text {even }}=0.30 \pm 0.04$ and $\sigma_{\text {odd }}=0.25 \pm 0.04$. A similar qualitative trend is found in the LSDA results, though quantitatively it is somewhat weaker. energy, (2) a substantial probability of non-trivial ground state spin, and (3) a significant even/odd effect in the addition energies. In comparison to LSDA, DMC typically predicts a somewhat larger spin gap; as a consequence, it has a tendency to find smaller ground state spins and a somewhat stronger even/odd effect in the addition spectra. These findings suggest that LSDA, as compared to DMC, in some sense over predicts the effect of interactions. It is interesting to note that a similar conclusion concerning overly strong interactions in LSDA was reached in the large dot regime ${ }^{25}$ using the Strutinsky analysis scheme.

Acknowledgments: We thank Weitao Yang for helpful discussions. This work was supported in part by the NSF (grants DMR-0103003 and DMR-0205328).
* Current address: Institut für Theoretische Physik, J.W.GoetheUniversität, Frankfurt am Main, Germany.

$\dagger$ Permanent address: Laboratoire de Physique Théorique et Modèles Statistiques (LPTMS), 91405 Orsay Cedex, France

${ }^{1}$ L. P. Kouwenhoven, C. M. Marcus, P. L. McEuen, S. Tarucha, R. M. Westervelt, and N. S. Wingreen in Mesoscopic Electron Transport, edited by L. L. Sohn, L. P. Kouwenhoven, and G. Schön (Kluwer, New York, 1997), pp. 105-214.

2 I. L. Aleiner, P. W. Brouwer, and L. I. Glazman, Phys. Rep. 358, 309 (2002).

3 J. A. Folk, C. M. Marcus, R. Berkovits, I. L. Kurland, I. L. Aleiner, and B. L. Altshuler, Physica Scripta T90, 26 (2001).

${ }^{4}$ L. P. Kouwenhoven, D. G. Austing, and S. Tarucha, Rep. Prog. Phys. 64, 701 (2001).

5 M. Ciorga, A. S. Sachrajda, P. Hawrylak, C. Gould, P. Zawadzki, S. Jullian, Y. Feng, and Z. Wasilewski, Phys. Rev. B 61, R16315 (2000).

${ }^{6}$ F. Bolton, Phys. Rev. B 54, 4780 (1996).

7 J. Shumway, L. R. C. Fonseca, J. P. Leburton, R. M. Martin, and D. M. Ceperley, Physica E 8, 260 (2000).

${ }^{8}$ F. Pederiva, C. J. Umrigar, and E. Lipparini, Phys. Rev. B 62, 8120 (2000); Phys. Rev. B 68, 089901 (2003); C. J. Umrigar, unpublished (2004).

9 A. Harju, S. Siljamäki, and R. M. Nieminen, Phys. Rev. B 65, 075309 (2002)

10 A. D. Güçlü, J.-S. Wang, and H. Guo, Phys. Rev. B 68, 035304 (2003).

11 K. Hirose and N. S. Wingreen, Phys. Rev. B 59, 4604 (1999).

12 S. M. Reimann and M. Manninen, Rev. Mod. Phys. 74, 1283 (2002), and references therein.

13 O. Bohigas, in Chaos and Quantum Physics, edited by M.-J. Giannoni, A. Voros, and J. Zinn-Justin (North Holland, Amsterdam, 1991) pp. 87-199.

14 M. V. Berry, J. Phys. A 10, 2083 (1977).

15 Ya. M. Blanter, A. D. Mirlin, and B. A. Muzykantskii, Phys. Rev. Lett. 78, 2449 (1997).

${ }^{16}$ I. L. Kurland, I. L. Aleiner, and B. L. Altshuler, Phys. Rev. B 62, 14886 (2000).

17 D. Ullmo and H. U. Baranger, Phys. Rev. B 64, 245324 (2001).
18 G. Usaj and H. U. Baranger, Phys. Rev. B 64, 201319(R) (2001); Phys. Rev. B 66, 155333 (2002).

${ }^{19}$ U. Sivan, R. Berkovits, Y. Aloni, O. Prus, A. Auerbach, and G. Ben-Yoseph, Phys. Rev. Lett. 77, 1123 (1996).

20 S. R. Patel, S. M. Cronenwett, D. R. Stewart, A. G. Huibers, C. M. Marcus, C. I. Duruoz, J. S. Harris, K. Campman, and A. C. Gossard, Phys. Rev. Lett. 80, 4522 (1998).

${ }^{21}$ S. Lüscher, T. Heinzel, K. Ensslin, W. Wegscheider, and M. Bichler, Phys. Rev. Lett. 86, 2118 (2001).

${ }^{22}$ S. Lindemann, T. Ihn, T. Heinzel, W. Zwerger, K. Ensslin, K. Maranowski, and A. C. Gossard Phys. Rev. B 66, 195314 (2002).

${ }^{23}$ H. Jiang, H. U. Baranger, and W. Yang, Phys. Rev. Lett. 90, 26806 (2003); Phys. Rev. B 68, 165337 (2003).

${ }^{24}$ H. Jiang, D. Ullmo, W. Yang, and H. U. Baranger, Phys. Rev. B 69, 235326 (2004).

25 D. Ullmo, H. Jiang, W. Yang, and H. U. Baranger, Phys. Rev. B 70, 205309 (2004).

${ }^{26}$ O. Bohigas, S. Tomsovic, and D. Ullmo, Phys. Rep. 223, 43 (1993).

$27 r_{s}$ is calculated at the level of DFT using $r_{s}=1 / \sqrt{\pi \bar{n}}$, where $\bar{n}$ is the weighted average electron density $\bar{n}=\int n^{2}(\mathbf{r}) d \mathbf{r} / N$. This definition is not unique for a finite system but gives a very good estimate (see Ref. 23 for details).

28 W. M. C. Foulkes, L. Mitas, R. J. Needs, and G. Rajagopal, Rev. Mod. Phys. 73, 33 (2001).

29 C.J. Umrigar, K.G. Wilson and J.W. Wilkins, Phys. Rev. Lett. 60, 1719 (1988); C. J. Umrigar in Quantum Monte Carlo Methods in Physics and Chemistry, edited by M. P. Nightingale and C. J. Umrigar (Kluwer, Dordrecht, 1999) pp. 129-160.

${ }^{30}$ C. J. Umrigar, M. P. Nightingale, and K. J. Runge, J. Chem. Phys. 99, 2865 (1993).

31 In our LSDA calculations, we used the Tanatar-Ceperley parameterized form for the exchange-correlation energy functional; see Ref. 23 for details.

32 The contribution of the Cooper channel, which should be included for time reversal invariant systems in a proper "universal Hamiltonian" description, is not taken into account here. 